\title{
BMJ Open Association between childhood allergic diseases, educational attainment and occupational status in later life: systematic review protocol
}

Laura Beate von Kobyletzki, ${ }^{1,2}$ Linda Beckman, ${ }^{2}$ Liam Smeeth, ${ }^{3}$ Martin McKee, ${ }^{3,4}$ Jennifer K Quint, ${ }^{5}$ Katrina Abuabara, ${ }^{6}$ Sinead Langan ${ }^{3,7}$

To cite: von Kobyletzki LB, Beckman L, Smeeth L, et al. Association between childhood allergic diseases, educational attainment and occupational status in later life: systematic review protocol. BMJ Open 2017;7:e017245. doi:10.1136/ bmjopen-2017-017245

\section{- Prepublication history and} additional material for this paper are available online. To view these files please visit the journal (http://dx.doi.org/10. 1136/bmjopen-2017-017245).

Received 11 April 2017 Revised 17 August 2017 Accepted 18 August 2017

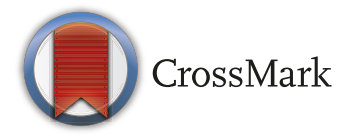

For numbered affiliations see end of article.

Correspondence to Dr Laura Beate von Kobyletzki; laura.von.kobyletzki@kau.se

\section{ABSTRACT}

Introduction Childhood allergic diseases may prevent affected children from achieving their academic potential. Potential mechanisms include absence from school due to illness and medical appointments. Experience of symptoms in classes or leisure time, and stigma associated with visible signs and symptoms, including skin disease, requirements for medication during school time or the need for specific diets, may also contribute to reduced educational attainment. Studies have investigated the association between specific allergic diseases and educational attainment. The aim of this study is to systematically review the literature on allergic diseases, educational attainment and occupational status, and if possible, calculate meta-analytic summary estimates for the associations.

Methods Systematic electronic searches in Medline, EMBASE, Cochrane, Cumulative Index to Nursing \& Allied Health Literature (CINAHL), PsycINFO and education Resources Information Center (ERIC); hand search in reference lists of included papers and conference reports; search for unpublished studies in clinical trial registers and the New York Academy of Medicine Grey Literature Report; data extraction; and study quality assessment (NewcastleOttawa Scale) will be performed.

Analysis Data will be summarised descriptively, and meta-analysis including meta-regression to explore sources of heterogeneities will be performed if possible. Ethics and dissemination Dissemination in a peerreviewed, open-access, international scientific journal is planned.

PROSPERO registration number CRD42017058036.

\section{BACKGROUND}

\section{Allergic diseases}

Asthma, eczema, allergic rhinitis and food hypersensitivity are important contributors to the global burden of disease, ${ }^{12}$ especially among children and adolescents, about $50 \%$ of whom are affected by at least one of these allergic conditions. ${ }^{3}$ There is evidence that these specific allergic diseases can have a substantial impact on quality of life compared with individuals in the general population. ${ }^{4-6}$
Strengths and limitations of this study

- Systematic review and meta-analysis of studies that have a study design that allows assessment of temporality.

- Will offer the highest level of evidence possible for informed decisions.

- Potential clarification whether the effect of allergic disease status on educational attainment and occupational status is modified by socioeconomic status.

- The range of different definitions of allergic disease status and different assessment of educational attainment and occupational status in the literature might hamper collation of outcomes.

\section{Academic attainment and allergic diseases}

Childhood allergic diseases may hinder academic achievement in several ways. (1) Children may miss school because of the physical effects of their conditions or the need to attend medical appointments. (2) Skin lesions requirements for medication during school time or specific diets might be associated with stigma affecting social interactions and performance at school. (3) Eczema is characteristically pruritic, leading to inadequate sleep and poor concentration. (4) Allergic rhinitis might also reduce concentration due to symptoms and the need for sedating medications that may impair performance. (5) In addition altered nutrient intake due to food hypersensitivity and bowel pain may impair academic attainment. ${ }^{7-9}$ (6) Finally, eczema and allergy have been linked to attention deficit hyperactivity disorder (ADHD), suggesting another possible pathway to lower educational attainment.

Educational attainment influences the wellbeing of children ${ }^{10}$ and influences future health, occupational and social opportunities. Other chronic illnesses, such as epilepsy 
and childhood cancer, have been shown to have a negative impact on education attainment. ${ }^{11} 12$ However, an initial scoping review has found variable findings in relation to educational attainment among those with asthma and food hypersensitivity. Thus, children with asthma have a higher rate of school absence but, despite this, some studies show only weak associations and others show no association between asthma and impaired educational attainment. A few studies have examined the employment of individuals who experienced asthma during childhood, finding that those who had asthma in childhood experience persisting disadvantage; specifically, persons with severe asthma were less likely to be employed as adults. ${ }^{13} 14$ Further, allergic rhinitis and antihistamine treatment have been associated with poor school performance, potentially due to the sedating effect of antihistamines, with lower grades observed in those taking examinations during seasons with peak rhinitis symptoms compared with during seasons where rhinitis is quiescent. ${ }^{15}$ A recent systematic review (SR) highlighted a dearth of studies focusing on eczema and academic attainment and occupational status; only one relevant population-based study was identified and this study did not support a relationship between eczema and reduced educational attainment. ${ }^{16}$

The relationship between allergic diseases and socioeconomic status (SES) is complex, with much scope for confounding and mediating factors that could influence any observed association between educational attainment and allergic diseases. There is a positive socioeconomic gradient in eczema, whereby risks of developing eczema are higher with higher maternal education. This association can be expected to promote higher academic attainment, potentially reducing any limitations related to having eczema. ${ }^{17}$ The data on associations with SES are mixed and may vary by allergy type. Some studies suggest that asthma, allergic rhinitis and food hypersensitivity may exhibit a negative socioeconomic gradient, whereby associations with lower maternal education might worsen any negative effect of disease on educational attainment. ${ }^{18}$

To the best of our knowledge, previous SRs have not assessed the relationship between allergic diseases and educational attainment.

The aim of this study is to investigate the association between specific allergic diseases, educational attainment and occupational status in adult life. Specifically, the following are our objectives:

1. to assess the associations between childhood asthma, allergic rhinitis, food hypersensitivity and eczema and educational attainment at any age during childhood and adolescence

2. to assess the associations between childhood asthma, allergic rhinitis, food hypersensitivity, eczema and highest academic degree

3. to assess the associations between childhood asthma, allergic rhinitis, food hypersensitivity, eczema and occupational status.
For objectives 1-3, we will assess if there is a relationship between study outcomes and severity of the allergic diseases. The study tests the hypotheses that asthma, allergic rhinitis, eczema and food hypersensitivity (particularly when severe) are related to lower educational attainment and reduced occupational status.

\section{METHODS}

An SR will be undertaken to evaluate the effects on (1) educational attainment during childhood and adolescence; and on (2) highest academic degree and occupational status during adulthood in children with

1. eczema (during childhood)

2. asthma (during childhood)

3. allergic rhinitis (during childhood)

4. food hypersensitivity (during childhood).

For each of these exposures, we will stratify the results by disease severity (where possible).

\section{Search strategy}

Systematic literature search in Medline, EMBASE, Cochrane, CINAHL, PsycINFO and ERIC, hand search in reference lists of included papers and conference reports will be performed. The abstracts included in the search that refer to conference reports will be assessed. Abstracts and conference proceedings will only be used to search for full publications that meet the inclusion/exclusion criteria. Unpublished studies will be searched by investigating clinical trial registers (such as ClinicalTrials.gov) and the New York Academy of Medicine Grey Literature Report (www.greylit.org).

Searches will also be performed on Google and Google Scholar search engines (see online supplementary appendix 1).

\section{Inclusion criteria}

- population-based studies with data on both the exposure (specific allergic diseases) and the outcome (educational attainment or occupational status) where temporality can be determined

- interventions: studies will not be selected based on treatment, although these data will be extracted for comparison purposes

- assessment of at least one of the following: (1) educational attainment from age 6 years until age 18 years, and (2) academic attainment in adulthood, for example, highest academic degree or occupational status

- studies that use a standardised definition of atopic disease and contain diagnosis of asthma, allergic rhinitis, eczema or food hypersensitivity

- no language restrictions will be used.

\section{Exclusion criteria}

We will exclude studies that focus exclusively on localised forms of eczema (ie, hand eczema) or studies that do not assess the outcomes of interest. 


\section{Endpoints}

\section{Primary endpoints}

- educational attainment at any age during childhood and adolescence, which could be measured by examination scores, grades or the highest level of educational attainment

- highest academic degree, for example primary school degree, high school degree, college degree, university degree or higher

- Occupational status based on standard registrar general, including not in education, employment or training status (where available) and salary (where available).

\section{Primary exposures of interest}

1. eczema

2. asthma

3. allergic rhinitis

4. food hypersensitivity.

Definitions of allergic diseases:

Physician-diagnosed or in accordance with validated diagnostic criteria.

\section{Potential confounding variables}

SES, age, sex, familial educational attainment, alcohol overconsumption by the individual, medications that may impair performance (eg, antihistamines, oral corticosteroids) and recreational drugs use will be considered as potential confounding variables. We will assess if sex or age of onset of specific allergic diseases is an effect modifier. We will also explore the impact of adjusting for sleep loss and ADHD (where available) as these potential covariates may be on the causal pathway between allergic diseases, and poor educational attainment and lower status occupation.

\section{Eligibility assessment}

Two reviewers will independently assess the eligibility of studies based on the title and abstract. If either of the reviewers considers a study potentially eligible, the full article will be retrieved for further assessment. In the second screening phase, full text papers will be assessed independently by the two reviewers using a standardised data extraction form designed to describe the characteristics of studies to be included as set out in the recommendations in the Cochrane Handbook section 5.1.0. Disagreements will be resolved by a third reviewer.

\section{Data extraction}

A data extraction form will be developed and piloted by reviewers on a random selection of included studies. Two reviewers will independently extract the data from included studies using this form. Extracted data items will include characteristics of each study, study methodological quality items and the outcomes of interest for each study.

\section{Study quality assessment}

Following the Cochrane Collaboration's recommendation to present potential biases for each study instead of using scores to rate quality, a set of quality appraisal items will be applied. These include biases in sample selection, validity of measures of disease and educational outcome, appropriateness of statistical analysis, and adjustment for confounders when applicable (see below).

Studies that do not assess temporality, for example cross-sectional studies, will be excluded.

The following tools will be used to assess study quality and for data extraction, obtained from the Cochrane Handbook resource and adapted for the current SR:

- 'List of study design features (table 13.2.a)', with exposure as intervention for collecting information about eligibility, and for collecting data about study design features

- data collection form for non-randomised studies

- Newcastle-Ottawa Scale for assessing the quality of a cohort study

- further quality assessment with Cochrane risk of bias tool.

\section{Statistical analysis}

Articles that meet the inclusion criteria will be recorded in table format in order to perform a systematic and narrative synthesis on the available evidence. We will study each allergic disease individually. If studies include combinations of different allergic diseases, we will seek to distinguish the separate effects; if this is not possible, we will report the combined data. If possible, we will stratify by disease severity of eczema, asthma or rhinitis. For allergic rhinitis, where possible, we will undertake sensitivity analyses restricted to hay fever season for educational attainment outcomes. We plan to use fixed effect models on ORs and test for heterogeneity (likely). Analyses are based on a causal model; and as we look at several possibly concurring risk factors, risk ratios will be assessed in models in which potential explanatory factors are added sequentially. Therefore, we plan to use fixed effects model meta-analysis if appropriate. If possible, we will undertake meta-analysis of study findings, including meta-regression, to explore sources of heterogeneities (eg, methodological quality, age of participants (children/adults), study duration, location and other differences due to confounding factors). ${ }^{19}$ Potential publication will be assessed by visual inspections of funnel plots. All statistical analyses will be performed with Stata MP 14 (by LBvK, SML) and for each outcome separately. Analyses will be performed separately for each disease outcome, and each disease outcome will be presented separately in the resulting publication.

\section{Causality assessment}

We will assess causality on the basis of Bradford Hill's nine criteria, paying particular attention to the following: 
1. the magnitude of association after adjusting for social class (strength of association)

2. the specificity of the finding for atopic disease versus other chronic diseases (if possible)

3 . the dose response effect according to disease severity

4. the consistency of association across different cultures and countries

5. the plausibility of the mechanism (as determined through the wider literature).

All studies included will be longitudinal providing evidence for temporality.

\section{Registration}

The protocol of the study was registered in Prospero. The manuscript reporting adheres to the Preferred Reporting Items for Systematic Reviews and Meta-Analyses Protocol guidelines. The completed checklist is included as an online supplementary file indicating the line/page numbers where items can be found.

\section{Author affiliations}

${ }^{1}$ Department of Dermatology, Lund University, Skåne University Hospital, Malmö, Sweden

${ }^{2}$ Department of Public Health Sciences, Karlstads Universitet, Karlstad, Sweden

${ }^{3}$ London School of Hygiene \& Tropical Medicine, London, UK

${ }^{4}$ Department of Health Services Research and Policy, London School of Hygiene \& Tropical Medicine, London, UK

${ }^{5}$ Respiratory Epidemiology, Occupational Medicine and Public Health, Imperial College London, London, UK

${ }^{6}$ Program for Clinical Research, Department of Dermatology, University of California, San Francisco, California, USA

${ }^{7}$ Department of Dermatology, St John's Institute of Dermatology, London, UK

Acknowledgements The authors acknowledge the help of Marika Svalstedt, librarian, with search strategies.

Contributors All authors participated in the concept and design of the study. LBvK and SL drafted the manuscript. LB, LS, MMK, JKQ and KA critically revised the manuscript for important intellectual content. All authors read and approved the final manuscript.

Funding SL is funded by a Wellcome Senior Fellowship in Clinical Science (205039/Z/16/Z). LS is funded by a Wellcome Senior Fellowship in Clinical Science (098504/Z/12/Z). KA is supported by NIH UCSF-CTSI Grant Number UL1 TR000004, the Dermatology Foundation and the Amos Medical Faculty Development Program.

Competing interests None declared.

Provenance and peer review Not commissioned; externally peer reviewed.

Data sharing statement Results will be disseminated in scientific peer-reviewed journals.

Open Access This is an Open Access article distributed in accordance with the Creative Commons Attribution Non Commercial (CC BY-NC 4.0) license, which permits others to distribute, remix, adapt, build upon this work non-commercially, and license their derivative works on different terms, provided the original work is properly cited and the use is non-commercial. See: http://creativecommons.org/ licenses/by-nc/4.0/ (c) Article author(s) (or their employer(s) unless otherwise stated in the text of the article) 2017. All rights reserved. No commercial use is permitted unless otherwise expressly granted.

\section{REFERENCES}

1. WHO. Global surveillance, prevention and control of chronic respiratory diseases: a comprehensive approach. $2007 \mathrm{http} / / / \mathrm{www}$. who.int/gard/publications/GARD\%20Book\%202007.pdf (accessed 3 Mar 2013).

2. GBD 2015 Disease and Injury Incidence and Prevalence Collaborators. Global, regional, and national incidence, prevalence, and years lived with disability for 310 diseases and injuries, 19902015: a systematic analysis for the global burden of disease study 2015. Lancet 2016;388:1545-602.

3. Ballardini N, Kull I, Lind T, et al. Development and comorbidity of eczema, asthma and rhinitis to age 12: data from the BAMSE birth cohort. Allergy 2012;67:537-44.

4. Lundberg $L$, Johannesson $M$, Silverdahl $M$, et al. Health-related quality of life in patients with psoriasis and atopic dermatitis measured with SF-36, DLQI and a subjective measure of disease activity. Acta Derm Venereol 2000;80:430-4.

5. Maksimović N, Janković S, Marinković J, et al. Health-related quality of life in patients with atopic dermatitis. J Dermatol 2012;39:42-7.

6. Goldney RD, Ruffin R, Fisher LJ, et al. Asthma symptoms associated with depression and lower quality of life: a population survey. Med $J$ Aust 2003;178:437-41.

7. Worldwide variation in prevalence of symptoms of asthma, allergic rhinoconjunctivitis, and atopic eczema: ISAAC. The International Study of Asthma and Allergies in Childhood (ISAAC) Steering Committee. Lancet 1998;351:1225-32

8. Boyce JA, Assa'ad A, Burks AW, et al. Guidelines for the diagnosis and management of food allergy in the United States: report of the NIAID-sponsored expert panel. J Allergy Clin Immunol 2010;126(6 Suppl):1105-18.

9. Cummings AJ, Knibb RC, King RM, et al. The psychosocial impact of food allergy and food hypersensitivity in children, adolescents and their families: a review. Allergy 2010;65:933-45.

10. Campbell FA, Ramey CT, Pungello E, et al. Early childhood Education: young adult outcomes from the abecedarian project. App Dev Sci 2002;6:42-57.

11. Black KC, Hynd GW. Epilepsy in the school aged child: Cognitivebehavioral characteristics and effects on academic performance. School Psychology Quarterly 1995;10:345-58.

12. Armstrong FD, Horn M. Educational issues in childhood cancer School Psychology Quarterly 1995;10:292-304.

13. Taras H, Potts-Datema W. Childhood asthma and student performance at school. J Sch Health 2005;75:296-312.

14. Milton B, Whitehead M, Holland $\mathrm{P}$, et al. The social and economic consequences of childhood asthma across the lifecourse: a systematic review. Child Care Health Dev 2004;30:711-28.

15. Walker S, Khan-Wasti S, Fletcher M, et al. Seasonal allergic rhinitis is associated with a detrimental effect on examination performance in United Kingdom teenagers: case-control study. J Allergy Clin Immunol 2007;120:381-7.

16. von Kobyletzki LB, Beckman L, Smirnova J, et al. Eczema and educational attainment: a systematic review. Br J Dermatol 2016:doi: 10.1111/bjd.15242. [Epub ahead of print 20 Dec 2016].

17. Uphoff E, Cabieses B, Pinart M, et al. A systematic review of socioeconomic position in relation to asthma and allergic diseases. Eur Respir J 2015;46:364-74.

18. Almqvist $\mathrm{C}$, Pershagen $\mathrm{G}$, Wickman M. Low socioeconomic status as a risk factor for asthma, rhinitis and sensitization at 4 years in a birth cohort. Clin Exp Allergy 2005;35:612-8

19. MOOSE guidelines for meta-analyses and systematic reviews of observational studies. https://www.editorialmanager.com/jognn/ account/MOOSE.pdf (accessed 7 Mar 2017) 